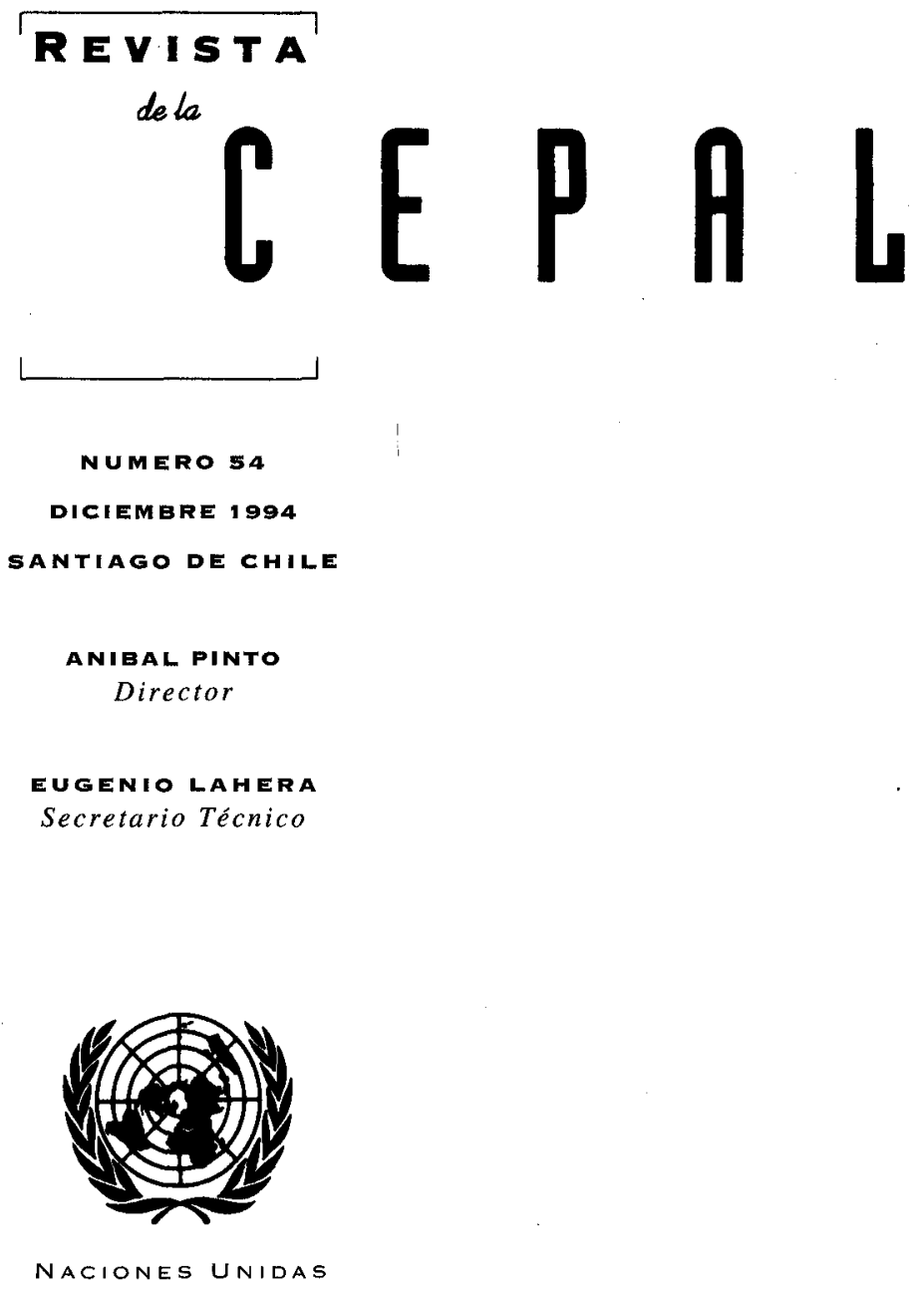


Mercados de salud: morfología, comportamiento y regulación

Jorge Katz y Ernesto Miranda

¿Liberalización o desarrollo financiero?

Günther Held

Ajuste fiscal y gasto social

Rossella Cominetti

Logros y obstáculos en la educación formal de las mujeres

Diane Alméras

¿Qué se entiende por flexibilidad del mercado de trabajo?

Ricardo A. Lagos

Modernización de la supervisión bancaria

Christian Larraín

Costos y beneficios de la integración centroamericana

Luis Cáceres

Algunas enseñanzas de las privatizaciones en Argentina

Daniel Azpiazu y Adolfo Vispo

Ventajas comparativas extraordinarias y crecimiento a largo plazo:

el caso de Ecuador

André A. Hofman y Rudolf Buitelaar

Industria cultural y nuevos códigos de modernidad

Martín Hopenhayn

Orientaciones para los colaboradores de la Revista de la CEPAL

Publicaciones recientes de la CEPAL 


\section{Modernización de la supervisión bancaria}

\section{Christian Larraín P.}

Asesor, Superintendencia de Bancos e Instituciones

Financieras, Chile
En este artículo se analizan los principales desafíos que enfrenta la modernización de la supervisión bancaria, a la luz de las actuales transformaciones del sistema financiero a nivel internacional y de las crisis bancarias recientes. Frente a un cuadro altamente dinámico — con fuerte desarrollo tecnológico en el ámbito de la informática; acentuada competencia al interior del mercado de capitales originada en procesos de liberalización; creciente protagonismo de los conglomerados financieros con el objeto de ganar economías de alcance, y una gran volatilidad de las principales variables económicas que acentúa mucho los riesgos financieros - la supervisión bancaria debe ser flexible para permitir el aprovechamiento de las oportunidades de mejorar la rentabilidad y eficiencia de las instituciones financieras. Pero a la vez debe adaptarse para garantizar el cumplimiento de sus objetivos estratégicos, a saber, dar estabilidad al sistema, otorgar transparencia y proteger a los avales estatales comprometidos. En este marco, los grandes temas de discusión en materia de supervisión bancaria en América Latina son: los estándares de adecuación del capital y el papel de este factor en la supervisión; los mecanismos de valorización del capital económico de un banco, a valor libro o de mercado; la forma de perfeccionar la cobertura de los riesgos financieros en la supervisión; la manera de incorporar la regulación de conglomerados financieros, y la importancia de adoptar un enfoque integral de supervisión, haciendo hincapié en la calidad de la gestión. 
I

\section{Introducción}

Las publicaciones financieras especializadas plantean que las características especiales de los bancos conducen a la necesidad de una regulación y supervisión públicas de su actividad. La regulación bancaria tiene como objetivo estratégico velar por la transparencia y estabilidad del sistema bancario, en un marco de eficiente asignación de los recursos financieros. Dicha regulación debe adoptar un enfoque dinámico, adecuándose permanentemente a las transformaciones en la forma de funcionamiento del sistema, con el objeto de garantizar el cumplimiento de su objetivo estratégico.

Las experiencias de crisis financieras en distintos países latinoamericanos durante los años ochenta condujo a importantes cambios en los enfoques de supervisión aplicados por los reguladores bancarios (Held y Szalachman, 1992). Sin embargo, a mediados de los años noventa, por el elevado dinamismo de la operación del sistema bancario a nivel internacional y la experiencia acumulada a partir de crisis bancarias recientes, como las del Banesto y el Banco Latino, se plantean nuevos desafíos. En efecto, factores como la fuerte competencia entre entidades bancarias y no bancarias en el mercado de capitales, el gran avance tecnológico en el ámbito informático, así como la globalización, abren oportunidades de negocios y de mejoramiento de la eficiencia y rentabilidad de las instituciones, pero obligan a adecuar la supervisión bancaria a un contexto de creciente complejidad.

Este artículo se propone señalar las distintas opciones en materia de políticas de regulación bancaria que se abren a las autoridades supervisoras para abordar los desafíos de hoy, marcando los principales lineamientos orientadores. En muchos de los temas no existen posiciones definitivas, por lo cual sólo se busca identificar los términos de la polémica y entregar algunas orientaciones de política, más que efectuar un análisis exhaustivo con propuestas definitivas.

\section{II}

\section{La labor de los organismos supervisores}

Por el hecho de que buena parte de sus pasivos son líquidos a la par, cualquier perturbación en un banco que pueda afectar la confianza de los depositantes puede traducirse en retiros masivos de recursos. Las características especiales de los bancos hacen que los riesgos que se derivan del quehacer específicamente bancario puedan perjudicar no sólo a sus propios accionistas sino también al resto del sistema y a la sociedad como un todo (riesgo sistémico). Cuando una institución bancaria muestra problemas de solvencia, sus dificultades, a ojos del público, son asociadas con otras instituciones, aunque ellas estén económicamente sanas. Esto puede ocasionar corridas bancarias que afecten a la economía en su conjunto, lo que en la conceptualización económica se entiende como una externalidad negativa. Lo anterior se agudiza fuertemente en el caso de los bancos grandes, por las desastrosas consecuencias que podría tener su quiebra para el resto del sistema ${ }^{1}$.

Debido a la importancia de la fe pública en el funcionamiento del sistema bancario, y por la necesidad de otorgar confianza y evitar corridas bancarias, existe un seguro de depósitos (explícito e implícito).

El mayor problema que se deriva de este seguro es el llamado riesgo moral: es decir, la existencia del seguro eleva los incentivos para correr riesgos, lo que termina incrementando la probabilidad de tener que recurrir al seguro. Dado que los depositantes asegurados saben que no sufrirán pérdidas si el banco quiebra, ellos no vigilarán su comportamiento ni lo disciplinarán, ya sea retirando sus depósitos o incrementando el premio exigido por riesgo si la institución corre peligros excesivos (Mishkin,1992). En consecuencia, los bancos con seguro de depósitos pueden asumir más riesgos que los que correrían si no lo tuvieran (excepto en el caso de que exista una prima diferenciada por riesgo).

\footnotetext{
1 En otras palabras, son demasiado grandes para que se les deje quebrar.
} 
Un segundo problema asociado al seguro de depósitos resulta del efecto de selección adversa del tipo de empresarios que son atraídos para invertir en el sector bancario ${ }^{2}$. El valor del seguro de depósitos para un banco aumenta con la cantidad de riesgo que toma, ya que normalmente el costo del seguro no está ajustado en función de ello. Esta franquicia, entonces, es más valiosa para un empresario proclive al riesgo que para uno conservador. Ya que los depositantes asegurados tienen pocas razones para imponer disciplina a los bancos, los empresarios proclives al riesgo se sentirán atraídos a operar en la industria bancaria.

El papel que desempeña la información es otro rasgo importante del mercado bancario. Por ejemplo, si se piensa en los pequeños ahorrantes, se comprobará que el monto de los recursos con que operan no les permite, o no les justifica, el costo de analizar los volúmenes de información necesarios para evaluar a cabalidad el espectro de instituciones en las cuales pueden depositar sus fondos, con el fin de cautelarlos adecuadamente. De no existir un ente que les asegure la confiabilidad del sistema, o al menos les entregue la información con algún grado mayor de procesamiento para su comprensión, esta proporción de depositantes no participará y sus recursos derivarán a usos alternativos que, probablemente, no sean los mejores para el país. Una adecuada regulación que promueva la transparencia dará confianza a estos depositantes y evitará el expediente extremo del apoyo patrimonial del Estado.

De este modo, en el mercado de capitales existen importantes asimetrías de información, por lo que se requiere una adecuada regulación y supervisión de la actividad con el fin de evitar abusos de información y conflictos de intereses dentro del sistema financiero. Aparte involucrar un elemento de injusticia, estas conductas pueden llevar a un debilitamiento de la fe pública en el fun- cionamiento de estos mercados, con efectos perturbadores tanto para su eficiencia como para su estabilidad.

En resumen, las características especiales de funcionamiento del sistema bancario causan determinadas imperfecciones, como externalidades o asimetrías de información, que apuntan a la necesidad de regulación y supervisión estatales con el objeto de minimizar impactos potenciales sobre el seguro de depósitos y evitar conflictos de intereses asociados a tales asimetrías.

Esto debe hacerse en un marco de eficiente asignación de los recursos financieros, lo que implica garantizar una competencia leal al interior del mercado de capitales, sin la acción de subsidios cruzados. En el caso de los bancos, ha de evitarse que las prerrogativas de que gozan por el acceso a la red de seguri$\mathrm{dad}^{3}{ }^{3}$ se extiendan a las filiales o empresas coligadas que se ocupan de los negocios no bancarios.

En un contexto dinámico, lo anterior plantea el mayor desafío a la política pública en materia de regulación bancaria, ya que no sólo se requiere que haya intervención pública, sino también que se optimice el carácter de dicha intervención. La regulación bancaria y las prohibiciones tienen costos explícitos e implícitos, asociados tanto al uso de recursos necesarios para cumplir con ellas como a las oportunidades perdidas al desviar recursos a actividades ineficientes. En la medida en que dichos costos superen lo requerido para otorgar estabilidad al funcionamiento del sistema bancario y para minimizar el impacto sobre el seguro de depósitos, habrá lugar para inequidades competitivas en relación con otros organismos no bancarios, fomentándose un proceso de desintermediación "artificial" (Federal Reserve of Cleveland, 1993).

Al tratar de optimizar la regulación, surge la necesidad de nuevos enfoques y políticas que complementen los utilizados tradicionalmente.

\section{III}

\section{La experiencia internacional}

A nivel internacional, cuatro son las principales tendencias que están afectando el sistema bancario: la desintermediación originada en desarrollos tecnoló-

\footnotetext{
2 También está el efecto de selección adversa de clientes, que deriva de las asimetrías de información existentes en el sistema bancario.
}

gicos, la acentuación de la competencia motivada por la liberalización, un mayor protagonismo de los

\footnotetext{
3 En la literatura financiera anglosajona se entiende por "red de seguridad" el conjunto de mecanismos regulatorios que permiten el acceso de las instituciones financieras al Banco Central como prestamista de última instancia y al respaldo otorgado por el seguro estatal de depósitos.
} 
conglomerados financieros y un agudizamiento de los riesgos financieros.

Los mejoramientos en las telecomunicaciones han posibilitado transacciones más baratas, fáciles y completas, abriendo nuevos mercados a nivel internacional. Los computadores proveen la capacidad de almacenar y manejar grandes cantidades de información que facilitan la materialización de las recompensas y riesgos de las distintas inversiones, en tanto que el avance en las comunicaciones ayuda a mantener bien informados a los agentes del mercado. Este proceso ha traído consigo una eficiente intermediación no bancaria basada en el abaratamiento de los costos de procesamiento de la información, generando una importante desintermediación bancaria (Moody's Investors Service, 1992).

Además, existen importantes procesos de liberalización inscritos en la tendencia hacia un sistema de banca universal: se ha permitido el acceso de entidades financieras no bancarias a actividades tradicionalmente desempeñadas por los bancos y se ha permitido a la vez el ingreso de bancos a actividades financieras no tradicionales, como corretaje de valores, servicios de asesoría financiera, administración de carteras, administración de fondos mutuos, seguros de vida y otras (Corbo, Donoso, Hernández, Rosende y Valdés, 1993). Esto también ha contribuido a acentuar la competencia dentro del mercado de capitales.

Los cambios tecnológicos y los procesos de liberalización han generado las condiciones para un creciente protagonismo de los conglomerados financieros en el mercado de capitales, guiados por el propósito de aprovechar las economías de escala y de alcance. ${ }^{4}$ Aunque la tendencia mundial apunta hacia una ampliación del ámbito de los negocios de la banca, no hay una visión homogénea respecto de las nuevas áreas que se abarcarían ni del tipo de arreglos institucionales con que esto se haría. Desde el punto de vista organizacional, lo que ha predominado ha sido un esquema de holdings financieros: las nuevas actividades se realizan a través de filiales o empresas coligadas, más que facultando a los bancos para realizarlas en un departamento dentro de ellos.
Por último, en los años ochenta creció la importancia del manejo de los llamados riesgos financieros, debido a una mayor volatilidad de los tipos de cambio, las tasas de interés y la inflación. A nivel del mercado, esto dio lugar a la aparición de un amplio abanico de productos "derivados", destinados a otorgar cobertura frente a tales riesgos (O'Connor, 1993).

Por otra parte, algunas experiencias de crisis bancarias recientes también muestran elementos que conviene consolidar cuando se busca modernizar la supervisión, toda vez que resaltan algunas carencias en la cobertura de riesgos, ante las cuales debiera reforzarse la vigilancia.

En el caso del Banco Latino, en Venezuela, aunque no hay datos definitivos, los antecedentes preliminares indican que las pérdidas ascenderían a 3000 millones de dólares. La corrida de los ahorrantes para retirar sus fondos puso en jaque a nueve bancos comerciales más. Para evitar el efecto dominó, el Banco Central de Venezuela se vio obligado a flexibilizar su política monetaria antiinflacionaria. No sólo bajó los encajes del $15 \%$ al $12 \%$, sino que tuvo que inyectar al sistema bancario, junto con el Fondo de Garantía de Depósitos, unos 3500 millones de dólares, no sólo para enjugar el costo del Banco Latino mismo, sino también para socorrer a los restantes bancos que se vieron en apuros. Los problemas de este banco ponen de relieve el influjo de los riesgos financieros y de la concentración crediticia en los resultados de un banco, así como claras deficiencias en los controles internos y los límites necesarios para cautelar un sano funcionamiento, en particular de sus operaciones en el exterior.

En el caso del Banesto, informes preliminares del Banco de España indican que las necesidades de saneamiento son del orden de los 3500 millones de dólares. La situación tuvo su origen en un crecimiento excesivo de las operaciones, proceso que no fue acompañado de políticas adecuadas de cobertura de riesgos, y que exhibió claras deficiencias en los sistemas de información y los controles internos. Los antecedentes expuestos revelan deficiencias en la calidad de la gestión en ambos bancos.

\footnotetext{
4 Existen eccnomías de alcance en la oferta cuando la producción conjunta genera costos inferiores a los de la producción por separado. Existen economías de ámbito en el consumo cuando el consu-
}

mo conjunto genera mayor bienestar al consumidor que el efectuado en forma independiente. 


\section{IV}

\section{Modernización de la supervisión:}

\section{el debate actual}

Se considera aquí que las tendencias asociadas a la dinámica del sistema financiero - principalmente la agudización de la competencia, el desarrollo tecnológico, la acentuación de los riesgos financieros y el protagonismo de los conglomerados-constituyen lo que suele llamarse tendencias pesadas o estructurales. Dichas tendencias abren un mundo de oportunidades, frente a lo cual la regulación debe ser flexible, otorgando a las instituciones financieras la posibilidad de aprovechar las ventajas de la mayor rentabilidad originada en los nuevos negocios, interna y externamente; las economías de alcance derivadas de la oferta y el consumo conjunto de servicios financieros; el mejoramiento de la eficiencia gracias a las nuevas tecnologías; y la cobertura frente a riesgos financieros por el uso de derivados. En este sentido, más que oponerse a tales tendencias, lo que corresponde es que la regulación y la supervisión bancarias se adecuen a dichas realidades, con el objeto de garantizar el cumplimiento de sus objetivos estratégicos de estabilidad, transparencia y protección del seguro de depósitos.

Cabe agregar que la competencia y la ampliación del giro bancario brindan importantes beneficios sociales, en la medida en que contribuyen a la provisión de más y mejores servicios financieros a menor costo, y permiten el aprovechamiento de las economías de escala y de ámbito propias del sistema financiero.

El hilo conductor de la regulación bancaria debe ser una supervisión prudencial, a fin de actuar antes de que los problemas se materialicen y conduzcan a eventuales corridas que puedan poner en peligro la estabilidad del sistema y los avales estatales comprometidos detrás del seguro implícito y explícito de los depósitos.

\section{Los requisitos de una supervisión prudencial}

La manera como se manejan las instituciones financieras en problemas cuando se aproximan a un punto de insolvencia tiene importantes connotaciones para la solidez de largo plazo y la viabilidad del seguro de depósitos, y para la estabilidad del sistema bancario mismo.
A medida que los bancos se aproximan a un punto de insolvencia económica, tienen cada vez menos que perder si se deciden por una estrategia agresiva y por inversiones de alto riesgo, con la perspectiva de recuperar rentabilidad. La supervisión es una importante herramienta para controlar los incentivos perversos que enfrentan los bancos subcapitalizados.

En términos generales, la capacidad de los órganos supervisores para tomar medidas de corrección tempranas y efectivas depende de tres factores (Estados Unidos, Departamento del Tesoro, 1991). Primero, deben ser capaces de identificar los problemas potenciales antes de que ellos generen pérdidas para el seguro de depósitos. Segundo, si han identificado el problema, deben tener facultades para forzar las correcciones o para prevenir un ulterior deterioro. Finalmente, si el problema ha sido identificado y existen facultades del regulador, no deben titubear en usar su autoridad en la forma pertinente.

En general, el proceso de supervisión permite identificar los bancos que podrían crear pérdidas al seguro de depósitos. Sin embargo, en algunos casos los problemas - de niveles de capital o de calificación- no son plenamente detectados. Los bancos, por su parte, pueden "errar" en efectuar las adecuadas provisiones frente a las pérdidas esperadas, debido a un excesivo optimismo o a una deliberada mala representación.

Es posible que el proceso de examen de un banco no logre identificar la verdadera situación de éste por tres razones. Primero, algunos problemas pueden aparecer repentinamente. Segundo, algunos bancos pueden no haber sido examinados con la frecuencia adecuada. Un banco primeramente identificado sólo como "banco con problemas"s y que colapsa menos de seis meses después podría indicar que no fue examinado con la frecuencia requerida. Tercero, los exámenes pueden fallar en descubrir la magnitud de los problemas.

\footnotetext{
5 En los Estados Unidos el organismo de supervisión competente -Office of the Comptroller of the Currency (OCC) - califica internamente a los bancos con una calificación de 1 a 5 . La categoría de bancos con problemas recibe el número 3. Esta categoría, si bien significa un desempeño defectuoso, no permite presumir una pronta insolvencia de una institución financiera.
} 
Sin una adecuada detección de los problemas, la posibilidad de adoptar medidas correctivas tempranas frente a los bancos subcapitalizados es muy limitada.

Por otra parte, la capacidad de los órganos supervisores para identificar problemas es de limitada utilidad sin una adecuada autoridad para forzar acciones correctoras. Corrientemente, cuando la condición de un banco comienza a deteriorarse, las agencias bancarias disponen de un abanico de opciones para corregir el problema. Por ejemplo, después de cada inspección, los examinadores pueden reunirse con la administración, incluido el directorio, para discutir el funcionamiento del banco. Estas discusiones informales son a menudo suficientes para corregir problemas menos graves.

En casos más graves se puede, entre otras medidas, recurrir a planes de recapitalización, impedir transferencias de fondos fuera del banco, limitar los riesgos de ciertas operaciones, restringir el pago de dividendos, limitar el crecimiento, cambiar los administradores o congelar las operaciones del banco.

Es importante señalar que a menudo los organismos supervisores están dotados formalmente de autoridad para tomar medidas correctoras en bancos con problemas, pero las pruebas requeridas en los procedimientos administrativos deben ajustarse a estándares tan exigentes, que en la práctica les es muy difícil obligar a bancos cuyas actividades son legales y rentables, que mantengan más capital o apliquen políticas más prudentes en ciertos ámbitos.

El tercer requisito para una supervisión prudencial es la voluntad de los órganos supervisores de usar su autoridad. Frecuentemente se alega que ellos tienen las herramientas adecuadas para controlar el riesgo de un banco, pero que carecen de los incentivos adecuados para emplearlos apropiadamente. Las razones esgrimidas son diversas.

Un primer elemento es que los reguladores tienden a ser "capturados" por la industria que regulan. Esto es, los reguladores están destinados a servir y promover la industria que ellos regulan. La perspectiva de futuros empleos en la industria regulada influye en el comportamiento algunas veces permisivo de los reguladores (Mishkin,1992).

Un segundo factor que puede afectar la voluntad del regulador es la fuerte influencia que pueden ejercer en ciertas situaciones los estamentos políticos, con el objeto de proteger a ciertos grupos sociales que podrían verse afectados con la crisis de un determinado banco; esto impide una acción adecuada de las agencias reguladoras.

Un tercer factor es que los órganos supervisores tienen incentivos para evitar una rápida intervención de bancos en situación crítica porque las insolvencias bancarias y las pérdidas consiguientes para el seguro de depósitos deterioran la imagen del organismo supervisor, que parecería no estar cumpliendo bien con su cometido. De acuerdo con esta visión, los supervisores preferirían no admitir la magnitud de los problemas, postergando así una solución definitiva. Esta es la hipótesis que plantea el semanario The Economist (1994) respecto del Banco Latino.

Para evitar problemas de incentivos que pueden llevar a los supervisores a ser permisivos frente a la necesidad de tomar medidas correctoras en bancos con problemas, y considerando los elevados costos que puede tener el aplazamiento de una intervención, algunos proponen que la supervisión se ajuste a reglas claras y obligatorias, y que se elimine la discrecionalidad.

A dicho enfoque se contraponen dos argumentos (Stiglitz,1993). En relación con el debate "reglas versus discrecionalidad", el primero sostiene que es imposible fijar reglas que se ajusten exactamente a las particularidades de toda situación. El segundo postula que con cualquier esquema de reglas se eleva la probabilidad de cometer dos tipos de errores: los bancos que no deberían ser cerrados lo son, y los bancos que deberían ser cerrados no lo son. Ajustar los estándares probablemente incrementaría un tipo de error, reduciendo el otro.

Una posible solución sería un régimen en el cual, en el curso normal de los acontecimientos, los bancos subcapitalizados enfrentaran una serie de presunciones que gatillaran la aplicación de medidas correctivas por el órgano supervisor, en función de la gravedad del problema. Las desviaciones de este comportamiento quedarían a discreción de ese órgano, pero acotadas dentro de un marco dado por la regulación.

\section{Reforzamiento del papel del capital en los in- centivos con que operan las instituciones}

La solvencia de las instituciones bancarias depende de un conjunto de factores que pueden agruparse en el tradicional modelo CAMEL $^{6}$ (Larraín,1993). Sin embargo, casi por definición podemos decir que una dotación adecuada de capital constituye el factor determinante de la solvencia de los bancos. La conexión entre un capital inadecuado y la insolvencia es obvia,

6 Este modelo considera el capital, los activos, la gestión, las utilidades y la liquidez que, expresados en inglés (capital, assets, management, earning, liquidity) corresponden a la sigla CAMEL. 
puesto que se reconoce que un banco se hace insolvente sólo cuando su capital se reduce peligrosamente o desaparece.

Los beneficios de una adecuada dotación de capital pueden resumirse así: ${ }^{7}$

i) Menor probabilidad de insolvencia bancaria. Mientras más capital tiene un banco, mejor puede afrontar pérdidas inesperadas sin llegar a ser insolvente, dando tiempo al regulador para actuar prudencialmente.

ii) Menos incentivos para tomar riesgos. Los accionistas con poco capital comprometido tienen incentivos para tomar excesivos riesgos ("si el negocio es bueno tengo mucho que ganar, si el negocio es malo pierde el banco"). A medida que un banco compromete su capital a niveles cercanos a la insolvencia, los incentivos perversos se acentúan.

iii) Reducción de la exposición del seguro de depósitos. Cuando un banco cae en insolvencia, cada peso de pérdida que absorbe el capital es un peso menos que asume el patrimonio público.

A pesar de lo anterior, no se trata de llevar las exigencias de capital a niveles tales que puedan proteger a los bancos frente a cualquier choque sistémico: esto haría no viable el negocio bancario. Por otra parte, el rol de la supervisión prudencial es justamente evitar que las situaciones catastróficas se produzcan. En consecuencia, la solución es más compleja que una simple elevación general de las exigencias de capital.

Reforzar el papel del capital en la supervisión supone lo siguiente:

i) Adaptar los estándares de capital medidos como una relación fija de endeudamiento a otros flexibles, cuyos requerimientos estén en función del riesgo de los activos, por las líneas del Acuerdo de Basilea (Larraín y Zurita,1993; Banco de Pagos Internacionales,1988). Un esquema de esta naturaleza busca hacer que los costos de capital sean sensibles al riesgo de los activos de las instituciones financieras. La evidencia empírica (Avery y Berger,1990) muestra que un sistema de exigencias de capital en función del riesgo de origen de los activos es superior a uno de exigencias fijas, desde el punto de vista de la prevención de insolvencias bancarias.

\footnotetext{
7 Véase Estados Unidos, Departamento del Tesoro,1991.
}

ii) Diferenciar la carga de la supervisión en función del respaldo de capital. La idea es que la carga de la supervisión sea creciente a medida que ese respaldo se deteriora. Con esto - como con la aplicación de zonas de capital en Estados Unidos- se pretende que la batería de instrumentos regulatorios de que dispone el supervisor bancario se vaya gatillando según el grado de debilitamiento de la posición de capital del banco (Federal Reserve of Cleveland,1993).

iii) Elevar las exigencias de capital para efectos de la apertura a negocios fuera de la órbita bancaria tradicional. La idea es incrementar los requerimientos de capital sólo para la apertura a nuevos negocios.

Dos son los principales argumentos que sustentan este planteamiento. Primero, hay riesgos provenientes del poco conocimiento de las nuevas operaciones y de la escasa experiencia en su supervisión. El ejemplo más claro de esto es el de las fíliales que operan en el exterior. La mayoría de los países latinoamericanos carece de tradición en materia de operaciones bancarias en el exterior, lo cual involucra mayores riesgos que es necesario prevenir. En consecuencia, cualquier proceso de mayor apertura hacia actividades internacionales pasa por instituciones bancarias de alta solvencia, capaces de enfrentar mejor los riesgos involucrados en dichas operaciones.

Además, en la región es difícil apoyarse para estas operaciones en la capacidad de los organismos supervisores, ya que no tienen gran experiencia con este tipo de actividades (distinto es el caso de operaciones dentro de la Comunidad Europea o de Estados Unidos). Esto también incrementa el riesgo asociado a un proceso de internacionalización, que se aminora con bancos mejor capitalizados.

Segundo, hay riesgos provenientes del incremento del riesgo sistémico. El riesgo sistémico surge si una determinada insolvencia de unos pocos grandes deudores pone en peligro el sistema como un todo. Al ampliarse la esfera de actividades de los bancos mediante la apertura de filiales es posible que i) se incremente el riesgo sistémico, ya que en una quiebra aparece involucrado un conjunto mayor de actividades y ii) se eleve el costo social de mantener la estabilidad del sistema ante choques disruptivos.

Aun cuando los seguros estatales explícitos puedan estar acotados a la casa matriz de un banco, los problemas de una filial podrían contagiar al banco mismo. En efecto, si el mercado percibe una asociación entre el banco y sus filiales -especialmente si hay pocas limitaciones para compartir activos intangibles y tangibles-, es evidente que en caso de problemas de una filial el banco tendrá incentivos para 
aplicar una política de salvataje. Esto eleva la exposición de los avales estatales comprometidos detrás de la operación de los bancos y lleva a una extensión de la "red de seguridad".

Los principales argumentos en contra de mayores exigencias de capital para la apertura a nuevas áreas de negocios provienen de su efecto potencial en la competitividad internacional. Si bien en el corto plazo los mayores requerimientos de capital podrían afectar negativamente la competitividad de la banca por el deterioro ocasionado a su rentabilidad, lo sucedido en países desarrollados indica con toda claridad que, a la larga, la solvencia tiene una importante correlación positiva con la rentabilidad y la valorización accionaria de los bancos.

En efecto, estudios de bancos internacionales calificados por la evaluadora de riesgo IBCA muestran que existe una correlación estadística positiva entre el Indice de Cook y la rentabilidad, lo mismo que entre dicho índice y la valorización accionaria (Kredietbank,1993). Los mismos estudios muestran que el mecanismo a través del cual opera el efecto anterior es la reducción del costo de los fondos a los que acceden los bancos más solventes. La razón es que los inversionistas ponderan la relación riesgo/rentabilidad asociada a cada banco, exigiendo un menor premio por riesgo a aquellos bancos más solventes.

A los mismos resultados llega el estudio que contiene la propuesta del Departamento del Tesoro al Congreso de los Estados Unidos, destinada a modernizar la banca de dicho país. En ese estudio se pone de relieve que los bancos estadounidenses más exitosos internacionalmente coinciden con aquellos más solventes, por lo cual dicha propuesta sugiere la creación de la llamada Zona 1 para los bancos mejor capitalizados, a los cuales se otorgaría mayor liberalidad regulatoria.

\section{3. ¿Valorización libro o a precios de mercado?}

Un enfoque de supervisión prudencial con énfasis en el papel del capital apunta a que se intervenga antes de que el valor neto de un banco llegue a cero o se torne negativo, para no generar pérdidas que, en definitiva, pueden recaer sobre el seguro estatal de depósitos. Para estos efectos, es esencial que el valor económico o el capital de un banco estén adecuadamente medidos (Stiglitz, 1993).

Existe una controversia respecto de los criterios de medición de los activos y pasivos de un banco. Bajo los principios de contabilidad generalmente acep- tados, los requerimientos de capital se calculan sobre la base de costos históricos. La valoración a valor libro (o histórico) genera un sesgo sistemático, ya que los activos que están subvalorados respecto de su valor de mercado pueden ser vendidos para realizar las ganancias, mientras que aquellos que están sobrevalorados pueden ser mantenidos en los libros para no reconocer las pérdidas (Mishkin,1992).

Una valoración generalizada a precios de mercado permitiría a los reguladores saber rápidamente si en un banco el capital está cayendo bajo los niveles permitidos; de esta manera podrían aplicar medidas correctivas antes de que el valor neto del banco fuese cero. Con ello se reducirían las pérdidas para el seguro de depósitos y habría menos incentivos para aplicar una estrategia de alto riesgo a medida que el banco se deteriorara.

A pesar de su superioridad teórica, sin embargo, la valoración generalizada a precios de mercado tiene importantes problemas desde un punto de vista práctico. Dado que no hay mercados secundarios para gran parte de los activos y pasivos de las instituciones bancarias, los valores de mercado deberían ser estimados usando alguna forma de análisis de flujo de caja actualizado. La subjetividad inherente a tales procedimientos reduce la comparabilidad de las estimaciones efectuadas por las distintas instituciones, dificultando el trabajo de supervisión. Tales problemas favorecerían una mayor manipulación, incrementando la incertidumbre respecto de la verdadera condición de las instituciones, cuya viabilidad depende fuertemente de la confianza pública (Estados Unidos, Departamento del Tesoro,1991).

Además, el costo actual de desarrollar y aplicar una valoración generalizada a precios de mercado puede ser alto, con lo cual se verían afectadas las instituciones de menor tamaño.

Por las razones señaladas, hacer hoy una valoración generalizada a precios de mercado es asunto complejo, aunque este enfoque debiera ir teniendo un papel creciente. Por un lado, medir los cambios en los precios de mercado debido a modificaciones de tasas no presenta mayores dificultades. Por otro, cuando los cambios se originan en el riesgo crediticio de los activos la situación es más compleja, debiendo recurrirse a un esquema de provisiones en función de una estimación de las pérdidas esperadas de dichos activos. Este sistema permite una apropiada evaluación del valor económico de un banco, sin que para hacerla sea necesario incurrir en costos exagerados respecto de los beneficios. Por lo demás, es factible mejorar 
la periodicidad de la supervisión, de manera de tener un cuadro permanentemente actualizado de la condición de los bancos.

Por último, es preciso reconocer las imperfecciones en la medición del valor neto de un banco al momento de establecer los estándares regulatorios. Esto significa que si no se puede determinar con exactitud el capital económico de un banco, los requerimientos de adecuación del capital deberán ser lo suficientemente exigentes como para dejar un cierto margen de holgura que permita actuar prudencialmente aun en el caso de que las mediciones no sean exactas (Stiglitz,1993).

\section{El manejo de los riesgos financieros}

Producto de un escenario internacional caracterizado por una creciente volatilidad de las tasas, desde mediados de los años setenta las pérdidas originadas en cambios inesperados en las tasas de interés o tipos de cambio han llegado a ser un problema cada vez mayor para las instituciones financieras.

Tales pérdidas ocurren, por ejemplo, cuando alzas inesperadas de las tasas de interés deterioran el valor de mercado de los activos en mayor medida que el de los pasivos. Este cambio diferencial en las valoraciones de mercado ocurre si los activos de las instituciones son menos sensibles que sus pasivos (Kaufman,1984). En definitiva, toda variación de las tasas puede influir en las ganancias netas y la rentabilidad del banco, dependiendo de su exposición al riesgo, dada por un manejo más o menos eficiente de sus activos y pasivos.

Los problemas asociados a los riesgos financieros son bastante conocidos, pero no es fácil medir adecuadamente la exposición al riesgo. $Y$ sin tal medición no se puede efectuar la gestión de tales riesgos, lo que ha subrayado la importancia de acceder a metodologías adecuadas para su cobertura.

En esta perspectiva, los reguladores internacionales ligados al Acuerdo de Basilea han estado examinando la posibilidad de agregar a los requerimientos de capital un componente referido a los riesgos financieros (Banco de Pagos Internacionales, 1993). Se trata de un enfoque distinto al que predomina en la actualidad, según el cual la cobertura de los riesgos financieros se maneja fundamentalmente mediante la fijación de límites. En la propuesta que está en elaboración, la idea es exigir mayor capital a medida que se incrementa el riesgo financiero. Cabe recordar que los estándares internacionales de capital están deter- minados en función del riesgo crediticio y no del financiero.

A diferencia de los límites, los requisitos de capital brindan a los bancos mayores incentivos para recurrir a técnicas de cobertura, a la vez que garantizan la disponibilidad de un "colchón" de capital prudente para absorber pérdidas inesperadas. Pese a haber dificultades para su implementación práctica, avanzar en la aplicación de modelos que permitan una medición más rigurosa de los riesgos financieros tiene claras ventajas, ya que los problemas prácticos pueden superarse aplicando versiones más simplificadas de las metodologías existentes hoy para medir tales riesgos. Es posible que sea necesario combinar los requisitos de capital con la mantención de límites, como medio tanto para imponer topes absolutos a los riesgos de los bancos como para reforzar los controles internos.

\section{La regulación de los conglomerados}

A nivel internacional se tiende a ampliar el ámbito del negocio bancario hacia actividades fuera del giro tradicional. La producción integrada de servicios financieros tiene como principal objetivo aprovechar las economías de alcance.

La ampliación del giro bancario tiene, sin embargo, costos potenciales significativos: i) incremento potencial del riesgo sistémico; ii) agudizamiento de los conflictos de intereses, y iii) inequidades competitivas originadas en la acción de subsidios cruzados (Herring y Santomero, 1990).

El tema del riesgo sistémico fue tratado en una sección anterior, por lo que no cabe profundizar en él.

Los conflictos de intereses constituyen un problema que no deriva del respaldo estatal a algunos depósitos bancarios, sino que es común a todo el mercado de capitales y a muchos otros mercados (Valdés,1989). Mientras más amplio el abanico de servicios financieros ofrecidos por una firma, mayor la posibilidad de que aparezcan conflictos de intereses (Herring y Santomero,1990). Y puesto que los conglomerados participan en una gama mayor de transacciones, más amplia es su información.

Es posible que surjan conflictos de intereses cuando los bancos participan en negocios que, por su naturaleza, implican realizar inversiones por cuenta de terceros (Morandé y Sánchez,1992). En este caso los bancos, que también invierten por cuenta propia, podrían reservarse los mejores negocios para ellos y dejar el resto para las inversiones por cuenta de terceros. Asimismo, podrían anticipar transacciones con 
recursos de terceros, con miras a afectar los precios de mercado.

La preocupación por las inequidades competitivas proviene del temor de que un conglomerado financiero pueda usar -implícita o explícitamente- el acceso al Banco Central como prestamista de última instancia, o el menor costo de los fondos debido al seguro estatal de depósitos, para subsidiar otras líneas de negocios. Esto podría distorsionar la competencia con aquellas firmas que no tienen acceso a un apoyo similar.

Dado que la producción conjunta de servicios financieros se da principalmente a través de conglomerados (Valdés, 1989), la organización institucional de la banca pasa a ser fundamental para potenciar los beneficios sociales del proceso y para minimizar los costos asociados. En este sentido, la regulación es esencial para optimizar el resultado.

El propósito central de la regulación bancaria debe ser proteger el banco en forma tal que: i) la red de seguridad -que incluye el acceso al Banco Central como proveedor de liquidez y el seguro estatal de depósitos - no sea extendida a actividades en las cuales el expertise bancario sea irrelevante, ii) limitar los subsidios cruzados -implícitos o explícitos- del banco a sus filiales o empresas coligadas y iii) evitar los conflictos de intereses y los abusos por posesión de información privilegiada (Estados Unidos, Departamento del Tesoro, 1991). Esto no significa que deba haber una separación total respecto de todas las actividades no tradicionales del banco, ya que ello dependerá de la naturaleza de cada actividad (es decir, de su grado de complementariedad con las actividades tradicionales del banco, de los aprovechamientos posibles de economías de ámbito, de los potenciales conflictos de intereses involucrados y del costo de la regulación y la supervisión).

Las nuevas actividades que emprenda un banco pueden llevarse a cabo directamente (en un departamento del banco), o a través de empresas filiales o de empresas coligadas dependientes de un holding común. Estas distintas formas de organización determinan diversos grados de aislación legal, económica y de mercado del banco respecto de las nuevas actividades (Estados Unidos, Departamento del Tesoro, 1991).

Las diferentes combinaciones de separación legal y operacional dan lugar a tres modelos básicos para la organización de conglomerados financieros. ${ }^{8}$

\footnotetext{
${ }^{8}$ Aparte de estos modelos teóricos puros, existen otros que corresponden a distintas combinaciones de ellos.
}

\section{Modelo 1}

El modelo de integración completa — también llamado modelo de multibanca alemán - permite a los ejecutivos de un conglomerado conducir todas las actividades dentro de una sola entidad, de modo que con esta estructura se puede producir cualquier combinación de productos financieros con el mínimo costo. Sin embargo, la mayor eficiencia operativa de esta opción debe contrastarse con sus costos.

Los problemas ligados a prácticas oligopólicas, conflictos de intereses y choques disruptivos pueden exacerbarse en el esquema de multibanca. Además, existe en potencia un significativo campo para el uso de subsidios implícitos desde el banco hacia otras líneas de negocios.

Por áltimo, esta estructura es más difícil de regular que un banco que sólo desempeña las actividades tradicionales del giro. ${ }^{9}$ Sin separación operacional ni legal, la combinación de un amplio rango de actividades financieras presenta al regulador el desafío de entender las implicaciones de supervisión prudencial de un gran número de productos. El resultado puede ser una regulación costosa e imprecisa.

\section{Modelo 2}

En esta segunda estructura básica la función bancaria se ejerce en una casa matriz bancaria y las funciones no bancarias se realizan en filiales incorporadas separadamente. Corresponde al modelo británico, en el cual se inspira el esquema que actualmente se aplica en Chile.

La separación legal involucra algunos costos de eficiencia, por lo cual el costo de producir una combinación dada de servicios financieros puede ser algo mayor que en el caso anterior. Pero existen dos ventajas compensatorias. Primero, al separarse del banco las actividades no bancarias se facilita una supervisión equitativa tanto de los negocios ligados a bancos como de otros independientes que se desempeñan en el mismo giro, lo que reduce el costo de la supervisión.

Segundo, en principio, este modelo protege al banco de malos resultados en otras actividades emprendidas por el conglomerado, permitiéndole a la vez beneficiarse de los retornos positivos. Al tener obligaciones limitadas, las filiales no bancarias pueden aumentar pero no disminuir el valor del banco.

Esta ventaja puede ser ilusoria, sin embargo, si

\footnotetext{
$9 \mathrm{Al}$ analizar el modelo bancario germano, Kregel (1992) concluye que el factor más significativo no es la carencia de regulación que permite la banca universal, sino un enfoque distinto de la regulación.
} 
el banco considera que su reputación y el costo de sus fondos podrían verse afectados por la insolvencia de alguna filial, lo que creará incentivos para que el banco sostenga a esa filial incluso más allá de su inversión de capital. Si el mercado refuerza esta percepción, se abaratará el costo de los fondos del conglomerado, pero se distorsionará la competencia con aquellas actividades emprendidas por empresas no ligadas a un conglomerado bancario.

Para reducir este riesgo, las autoridades reguladoras pueden intentar el refuerzo de la separación legal con separaciones operacionales. El costo en eficiencia de esta medida es claro, pero la contribución al fortalecimiento de la disciplina de mercado de las filiales es menos cierta.

\section{Modelo 3}

Un tercer modelo es el del holding, que corresponde al de los Estados Unidos. Aquí la casa matriz es dueña tanto del banco como de las contrapartes no bancarias. Dado que la separación legal es mayor que en el modelo anterior, el costo de producir una combinación dada de productos financieros es probablemente algo más elevado en esta opción. Este modelo capta algunos de los beneficios sociales de las economías de alcance en la comercialización y distribución de productos financieros, con menos riesgo para la red de seguridad, al estar limitada su extensión más allá del banco; sin embargo, en la medida en que los cortafuegos inhiban la eficiencia operacional, habrá alguna pérdida de eficiencia.

Desde la perspectiva de la regulación, este modelo tiene sobre el modelo 1 las mismas ventajas que el modelo 2, pues facilita una supervisión equitativa y protege al banco. Pero la principal ventaja del modelo 3 respecto del 2 es que el banco puede tener menos incentivos para apoyar a una unidad no bancaria si se trata de una empresa coligada que si se trata de una filial del banco. En Estados Unidos la regulación ha tratado de acentuar esta separación requiriendo como cortafuegos que la empresa coligada difiera del banco en nombre, empleados, local y redes de distribución, con lo que se busca reducir el impacto negativo que la quiebra de una empresa coligada puede tener sobre la reputación del banco.

Evidentemente, dependerá de las condiciones particulares de cada país cuál modelo se ajusta mejor a él. Es esencial considerar la capacidad supervisora y la autonomía del organismo regulador. Asimismo, es importante la naturaleza de los vínculos entre los dis- tintos grupos del ámbito bancario y no bancario, $\mathrm{y}$ también la existencia de posibles avales estatales comprometidos en cada uno de ellos.

\section{Aplicación de un enfoque integral de supervisión}

Dada la creciente complejidad del modo de operación del sistema financiero, es preciso avanzar en la aplicación de un enfoque integral de supervisión. Para utilizar un esquema de supervisión prudencial, los organismos supervisores deben efectuar un permanente y sistemático análisis de los principales aspectos que determinan la situación de los bancos. Esto implica complementar los esquemas actuales de supervisión aplicados en los países latinoamericanos, que se basan fundamentalmente en una revisión de los activos y las provisiones de las instituciones financieras, y poner más el acento en elementos como la adecuación del capital y la evaluación de la gestión. En definitiva, se trata de avanzar en la aplicación de un enfoque de supervisión tipo CAMEL.

Es importante destacar aquí que la aplicación de este enfoque exige mucha coordinación e intercambio de información sobre los distintos aspectos de los bancos, dentro de los organismos de supervisión. Aunque para efectos analíticos sea posible diferenciar entre capital, activos, gestión, etc., en la práctica los distintos aspectos están muy relacionados entre sí. (Por ejemplo, una deficiente gestión puede llevar a problemas en la calidad de los activos o derivar en insuficiente capital).

Una de las áreas particularmente débiles de la supervisión bancaria en América Latina es la que corresponde a la evaluación de la gestión, a pesar de ser uno de los aspectos cruciales para proyectar la situación de las instituciones en el futuro, más allá de la radiografía que ofrecen los indicadores financieros de tipo cuantitativo.

En los Estados Unidos, en cambio, el organismo de supervisión pertinente, la OCC, pone en el centro de su análisis el tema de la calidad de la gestión. La premisa que sustenta todo el trabajo de la OCC es que la administración y el directorio del banco son los responsables últimos del funcionamiento de la institución. Para conocer mejor el peso relativo de las condiciones económicas externas y de factores de administración internos en el resultado de los bancos, ese organismo efectuó un estudio destinado a evaluarlos (OCC, 1988).

El estudio mostró que aunque las malas condiciones económicas hacen más difícil para un banco mantener un curso rentable, las políticas y procedimientos 
impulsados por la administración y el consejo directivo del banco tienen más influencia en el éxito o fracaso de la institución. En otras palabras, la mala gestión y otros problemas internos son el común denominador de los bancos insolventes o con problemas.

El manejo efectuado por la administración habría desempeñado un papel significativo en el deterioro de un $90 \%$ de los bancos insolventes o con problemas que la OCC evaluó. Muchas de las dificultades experimentadas por los bancos respondieron a inadecuadas políticas de préstamos, problemas en los sistemas de identificación de créditos, o debilidad en el cumplimiento de las políticas internas y de las regulaciones bancarias. En otros casos, el comportamiento imprudente del directorio o la administración se tradujo en prácticas riesgosas de otorgamiento de préstamos y en un excesivo crecimiento de ellos. Las prácticas de autobeneficio y fraude también fueron aspectos relevantes en el deterioro de más de un tercio de los bancos insolventes o con problemas.

La declinación económica contribuyó a las difi- cultades de muchos de los bancos evaluados. Específicamente, el factor económico fue un elemento significativo en un tercio de los bancos. Sin embargo, pocas veces fueron los factores económicos la única causa de declinación: sólo 7\% de los bancos insolventes o con problemas estaba libre de significativos problemas internos de gestión.

En definitiva, el estudio muestra que la administración y el consejo directivo son, a fin de cuentas, los responsables del éxito o el fracaso de un banco. Si en una institución financiera se establecen políticas, controles y sistemas sólidos cuando las condiciones económicas son buenas, aumentará su probabilidad de permanecer rentable en condiciones económicas adversas.

En un esquema de supervisión prudencial, los problemas de gestión identificados constituyen la base para la aplicación de medidas correctoras que apunten a solucionar los problemas de los bancos. Esto plantea la necesidad de que la supervisión bancaria en los países latinoamericanos examine más a fondo el aspecto de gestión.

\section{$\mathrm{V}$}

\section{A modo de conclusiones}

La creciente complejidad que caracteriza el modo de operación del sistema bancario en la actualidad, ofrece a las instituciones financieras un abanico de oportunidades para mejorar su eficiencia y rentabilidad, pero a la vez plantea a la supervisión bancaria una serie de desafíos en la tarea de garantizar el cumplimiento de sus objetivos estratégicos.

Esto queda de manifiesto al analizar experiencias de crisis bancarias recientes - como las del Banco Latino y el BANESTO - con el objeto de evitar o minimizar la ocurrencia de fenómenos de esa naturaleza.

Como premisas de un enfoque de supervisión prudencial es necesario que el organismo supervisor tenga la capacidad de identificar los problemas con rigor, y que cuente con la autoridad y voluntad para tomar medidas correctoras antes de que los problemas se materialicen.

Los siguientes son los principales temas en el debate actual en torno a la modernización de la supervisión bancaria: Primero, el fortalecimiento del pa- pel del capital, para lo cual es preciso adoptar estándares de adecuación del capital vinculados al riesgo de los activos, discriminar la carga supervisora en función del respaldo de capital y elevar los requerimientos de capital para la apertura a nuevas áreas de negocios. Segundo, el perfeccionamiento de los mecanismos de valoración, con un creciente énfasis en los precios de mercado por encima del valor libro. Tercero, la incorporación a las exigencias de capital de un factor destinado a la cobertura de riesgos financieros. Cuarto, la consideración en el esquema regulador de la presencia de conglomerados financieros, con el objeto de aprovechar los beneficios de las economías de alcance y minimizar los costos potenciales del riesgo sistémico, los problemas de conflictos de intereses y la acción de subsidios cruzados. Y quinto y último, el refuerzo de un enfoque de supervisión integral tipo CAMEL, que considere los distintos aspectos que determinan la condición de una entidad financiera, haciendo particular hincapié en el problema de la calidad de la gestión. 


\section{Bibliografía}

Avery, R. y A. Berger (1990): Risk-based capital and deposit insurance reform, Journal of Banking and Finance, $\mathrm{N}^{\circ} 15$.

Banco de Pagos Internacionales (1988): International Convergence of Capital Measurement and Capital Standards, Basilea, Suiza, Committee on Banking Regulations and Supervisory Practices.

(1993): The Prudential Supervision of Netting, Market Risks and Interest Rate Risks, Basilea, Suiza, abril.

Corbo, V., A.Donoso, L. Hernández, F. Rosende y S. Valdés (1993): El sistema bancario chileno: desarrollos recientes y sus perspectivas, Santiago de Chile, Universidad Católica de Chile, Instituto de Economía.

Estados Unidos, Departamento del Tesoro (1991): Recommendations for Safer, More Competitive Banks, Washington.

Federal Reserve of Cleveland (1993): Economic Commentary, Cleveland, Estados Unidos, marzo.

Held, G. y R. Szalachman (eds.) (1992): Regulación y supervisión de la banca, vols. 1 y 2, Santiago de Chile, Programa de las Naciones Unidas para el Desarrollo (PNUD)/CEPAL.

Herring, R. y A. Santomero (1990): The corporate structure of financial conglomerates, Journal of Financial Services Research, Kluwer Academic Publishers.

Kaufman, G. (1984): Measuring and managing interest rate risk: a primer, Economic Perspectives, Federal Reserve Bank of Chicago.

Kredietbank (1993): Monthly Bulletin, Bruselas, noviembre.

Kregel, J.A. (1992): Universal banking, US banking reform and financial competition in the ECC, BNL Quarterly Review, $\mathrm{N}^{\circ} 182$, septiembre.

Larraín, C. (1993): Adecuación de capital y regulación bancaria en
Chile, Información financiera, Santiago de Chile, Superintendencia de Bancos e Instituciones Financieras, agosto.

Larraín, C. y F. Zurita (1993): El Acuerdo de Basilea, Información financiera, Santiago de Chile, Superintendencia de Bancos e Instituciones Financieras,julio.

Mishkin, F. (1992): An evaluation of the treasury plan for banking reform, The Journal of Economic Perspectives, vol. 6, $\mathrm{N}^{\circ} 1$, Nashville, Tennessee, American Economic Association, invierno.

Moody's Investors Service (1992): Global Credit Analysis, Londres IFR Books.

Morandé,F. y J. M. Sánchez (1992): La expansión del giro bancario tradicional a los negocios no bancarios, La banca ante nuevos negocios y mercados, Santiago de Chile, Asociación de Bancos e Instituciones Financieras de Chile, Instituto de Estudios Bancarios.

OCC (Office of the Comptroller of the Currency, United States) (1988): An Evaluation of the Factors Contributing to the Failure of the National Banks, Washington.

O'Connor, S. (1993): Factores de desarrollo del mercado de derivados: la experiencia de Canadá, Monetaria, vol. XVI, No 2 , México, D.F., Centro de Estudios Monetarios Latinoamericanos (CEMLA).

Stiglitz, J. (1993): The role of the State in financial markets, World Bank's Annual Conference on Economics Development, vol. 2, Washington, D.C. Banco Mundial.

The Economist (1994): A stitch too late. Venezuelan banks, Londres, The Economist Newspaper, 12 de marzo.

Valdés, S. (1989): Regulación de los bancos y de sus servicios financieros afiliados, mimeo, Santiago de Chile. 